\title{
Light harvesting with metasurfaces: applications to sensors and energy generation
}

\author{
David T. Crouse · Eli Lansey • Isroel Mandel • \\ Ian Hooper
}

Received: 24 March 2014/Accepted: 10 August 2014/Published online: 6 September 2014

(C) The Author(s) 2014. This article is published with open access at Springerlink.com

\begin{abstract}
Metasurfaces have been receiving increasing interest due to the complex array of radiation controlling properties that are possible with single-layer films. This talk and work will focus on metasurfaces that can provide light filtering according to wavelength, polarization, and other properties of an incident beam, and applied to a variety of sensors. Also, metasurfaces that exhibit light trapping and localization that can be used for energy generation will be described. One last group of metasurfaces will be discussed that display complex dispersion curves that exhibit fast- and slow-light properties that can be used to study the complex electromagnetic phenomena.
\end{abstract}

\section{Introduction}

Composite engineered surfaces of various forms have been researched for years in many different technology fields (e.g., microwave, RF, optical) for applications that include

D. T. Crouse $(\square)$

Department of Electrical Engineering, The City College of New York, 160 Convent Avenue, New York, NY 10031, USA

e-mail: dcrouse@ccny.cuny.edu

E. Lansey

Riverside Research, 156 William St, New York, NY 10038, USA

I. Mandel

Department of Physics, The City College of New York, 160 Convent Avenue, New York, NY 10031, USA

\section{Hooper}

Electromagnetic and Acoustic Materials Group, Department of Physics and Astronomy, University of Exeter, Stocker Road, Exeter EX4 4QL, UK electromagnetic (EM) radiation filtering to determine the polarization state and/or wavelength of an incident beam, or to perform beam steering, light harvesting, and light concentrating [1-19]. This field has received increased attention recently because of the development of surface structures, composed of optically resonant metal structures (i.e., antennas) that operate in the infrared and visible spectral regions [20]. Yet a clear line that delineates what is, and what is not, a metasurface does not exist. From an applications perspective, and from the perspective of device engineers, such a delineation is artificial and irrelevant-what matters is designing a surface that performs a particular desired function. To be considered a true metamaterial or metasurface, it is often thought necessary that the structural feature sizes of the "meta-atoms" need to be smaller than $\lambda / 10$ where $\lambda$ is the wavelength of light to which the metamaterial is designed to respond. Restricting oneself to such materials and surfaces is very limiting and does not include the wide array of important EM phenomena that occur within photonic crystals, plasmonic structures, and other structures (e.g., the optical antenna arrays that are spurring a new generation of metasurface structures). With these structures, the feature sizes of the structural elements, the meta-atoms, may be approximately $\lambda / 4$ to $\lambda / 2$ in size, yet with these structures, one can perform a wide range of light-management functions, from complex polarization and wavelength filtering for advanced imaging systems, to light trapping and harvesting for renewable energy devices, light concentration to increase the sensitivity of detectors, and beam steering and forming. And with these structures being able to have EM properties that go well beyond what naturally occurring materials can achieve, they legitimately fit within a broader definition of metamaterials. This paper will review some of the work that our group at the National Science Foundation Industry/ 
University Cooperative Research Center for Metamaterials is doing on the development of metasurfaces.

In this paper, metasurfaces that perform photon sorting in the microwave spectral range will be reviewed and described, as well as engineered surfaces with which one can engineer the shape of surface plasmon dispersion curves.

\section{Photon sorting using waveguide cavity modes within patterned metal surfaces}

One particularly attractive light-management tool is the ability of a surface to sort EM radiation according to wavelength and to channel photons with different energies to different regions of a surface. The resulting sorted photons can then be individually absorbed, collected, or detected. Such a surface can be used for several important applications. One application is dual-wavelength IR discrimination and detection within a single small device. A second application is for renewable energy applications where the incoming solar radiation is split according to wavelength, and the different energy photons are channeled to different absorbers that collect the light and transfer the optical energy to electrical energy; this structure avoids the "thermalization losses" inherent in single-junction silicon solar cells.

With the devices developed in our works [21, 22], we aimed to have the engineered surfaces perform three functions: photon sorting, light localization, and absorption. Additionally, the devices were designed to perform these functions on incident light of any polarization and over a wide range of angles of incidence. As stated in [22], there has been some prior research on multi-band frequency selective surfaces (FSSs) to increase the capabilities of multi-frequency microwave antennas, yet these structures add discrete subreflectors that perform frequency-specific reflection and these additional discrete structures add weight and size to the antenna. Additionally, photon sorting has been performed using bimetallic nanoantennas and plasmonic gratings in the visible spectral regime. Yet these devices performed only one function, i.e., photon sorting, and often suffered from polarization dependence and angle of incidence dependence of the incident radiation, as opposed to the structures described in this paper.

In our work described in [22], a subwavelength sized aperture array (SAA) was designed to locally (i.e., near the surface) split microwave radiation according to frequency and to channel the light to differently sized apertures within each repeating unit cell and to individually absorb the photons within two non-overlapping spectral bands. The SAA was then fabricated and tested.

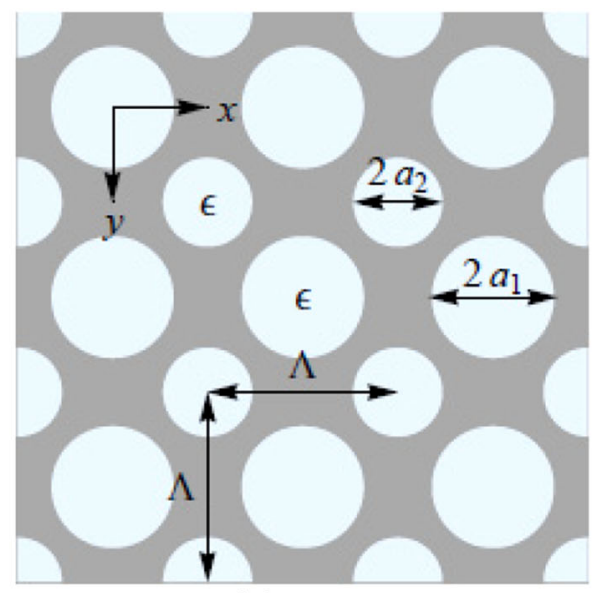

(a)

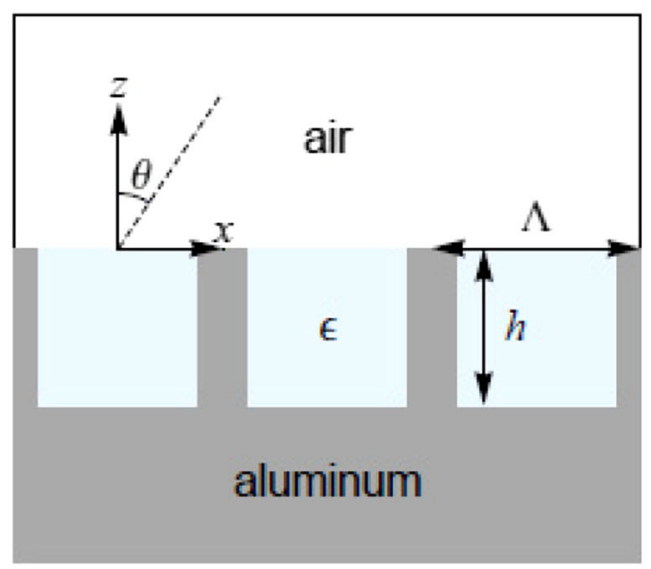

(b)

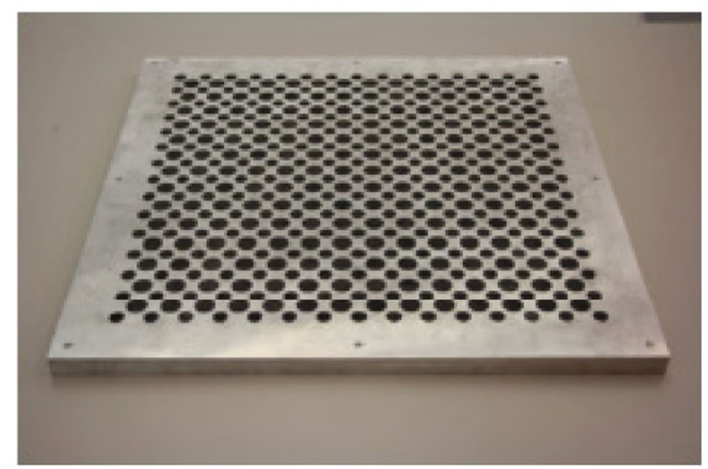

(c)

Fig. 1 A schematic of periodic cylindrical cavities in a metal is shown from top down (a), in a cross section through one set of cavities (b), and the final fabricated device (c). The gray region represents the metal, the light blue regions are the dielectric-filled apertures, and the white is the superstrate (air) above the cavities. Here, $\Lambda=26 \mathrm{~mm}, a_{1}=8.03 \mathrm{~mm}, a_{2}=5.74 \mathrm{~mm}, h=7 \mathrm{~mm}$, and $\theta$ is the angle of incidence [22]

Even though this particular structure was designed to operate in the microwave spectral region, the underlying principles can be applied to SAA that operate in other spectral regions [21]. To do this, one scales the geometric 
feature sizes of the structure and the wavelength of operation is correspondingly scaled (i.e., reducing all the dimensions of the physical features, say by one half reduces the wavelength of operation by one half). Of course, scaling the structure by a very large amount, say from the microwave to the visible spectral region, generally requires one to include the increased optical loss of the materials and also generally requires the use of different materials for the SAA.

But in the microwave, metals such as copper and aluminum are low loss. The structure that was designed in [22] is a compound cavity array. The cavities were not deeply subwavelength in size, and they were approximately $\lambda / 2 \sqrt{\varepsilon}$ where $\varepsilon$ is the complex dielectric permittivity of the material filling the cavities. Thus, this structure is not a pure metamaterial or metasurface that appears to be homogenous from the perspective of the incident radiation. The EM modes responsible for the SAA's properties are waveguide cavity modes (WCMs) that can be supported by the cavities. The photon sorting and light channeling themselves can be understood by considering the timereversed situation, because Maxwell's equations are timereversal invariant (with some important exceptions [22]). In the time-reversed situation, if one can excite the WCMs of different energies supported by differently sized cavities, then the WCMs will not be entirely confined to the cavity and will radiate out into free space, with the outwardly radiating energy diverging. This outwardly radiating diverging beam in the time-reversed frame of reference is an inwardly incident beam that converges to cavities in the forward-time frame of reference. This simple explanation describes how the light can experience the effects of the cavities prior to encountering any physical object (i.e., the metal surface); more detailed explanations can be found in [22].

The photon sorting structure is a two-dimensional square array of subwavelength cylindrical cavities embedded in aluminum. Each unit cell contains two cavities of different radii and identical heights, arranged in a rhombic lattice, see Fig. 1. The individual cavities within the unit cell are designed to support an effective-cavity resonance or cavity mode (CM) with amplified EM fields, where the lowest-order mode's frequency dependence is approximately given by [22]:

$f \approx \frac{c}{2 \pi \sqrt{\operatorname{Re}[\varepsilon]}}\left[\left(\frac{\pi}{h_{\mathrm{eff}}}\right)^{2}+\left(\frac{1.841}{a}\right)^{2}\right]^{1 / 2}$

where $a$ is the cavity radius and $h_{\text {eff }}$ is the effective height of the cavity (consisting of the physical geometric height plus some additional length as determined by the WCM's energy and mode order). Because the structure is to absorb the photons channeled to the two different apertures within

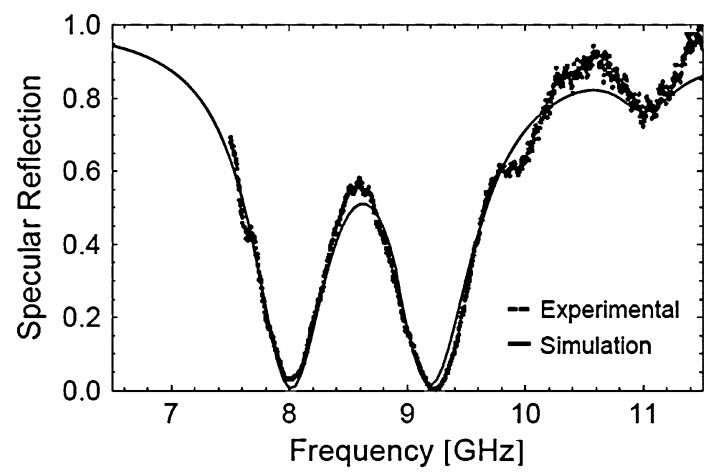

Fig. 2 Simulated and experimental specular reflection from the metasurface for $s$-polarized radiation at $\theta=17^{\circ}$ angle of incidence. The individual dips at 8.10 and $9.25 \mathrm{GHz}$ are due to the two different WCMs [22]

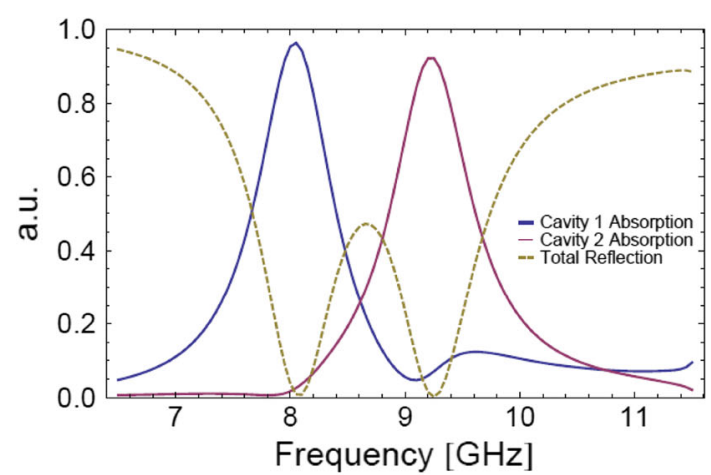

(a)

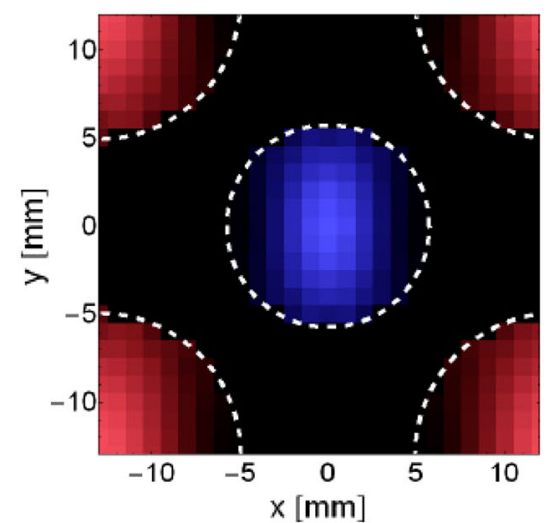

(b)

Fig. 3 a Simulated total reflection and the absorption in the two different cavities. It is seen that the structure performs efficient photon sorting and absorption. b A false-color plot of the volume loss density at a height of $3.5 \mathrm{~mm}$ inside the cavities at $8.10 \mathrm{GHz}$ (red) and $9.25 \mathrm{GHz}$ (blue) overlaid on the same plot [22]

each unit cell, an absorbing material was placed in the cavities. This material was a silicone elastomer dielectric (Sylgard 184) doped with $8.36 \%$ graphite; this resulted in a complex permittivity of $\varepsilon=4.33+0.22 i$. This value of $\varepsilon$ 

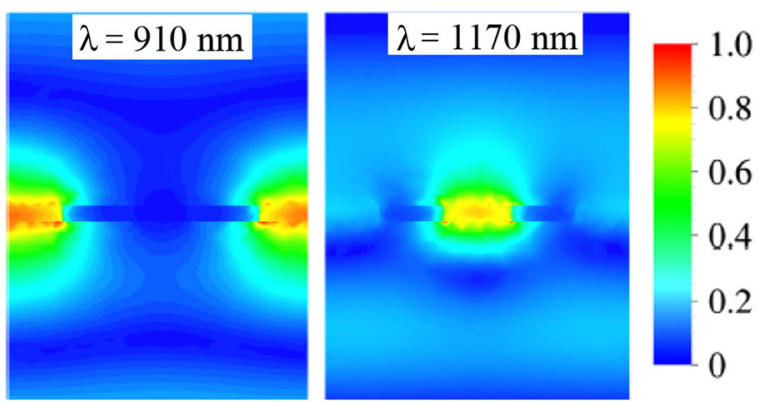

(a)

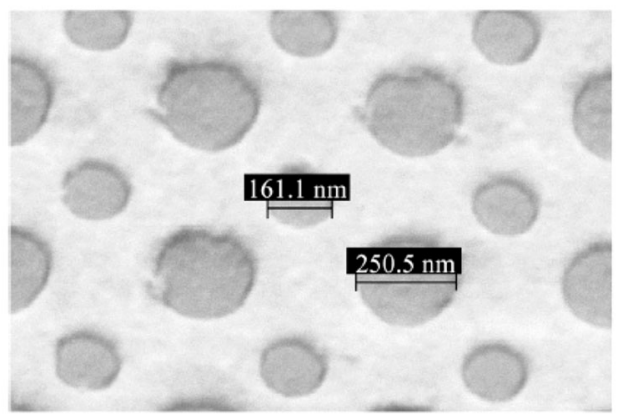

(b)

Fig. 4 a Fabricated structure (dimensions given in the text). b The simulated electric field magnitude for a normal incident beam of $\lambda=910 \mathrm{~nm}$ and $\lambda=1,170 \mathrm{~nm}$. The simulated results show that efficient photon sorting should occur, yet due to the more challenging fabrication of these small structures, the structural variations produce widening and shifts of the absorption peaks [21]

was carefully chosen to maximize concentration and achieve optimal coupling between the incident beam and the CM [23]. The two cavities were designed such that they support two non-overlapping WCMs that produce absorption bands at 8.10 and $9.25 \mathrm{GHz}$. To achieve this, the dimensions of the structure were $a_{1}=8.03 \mathrm{~mm}$, $a_{2}=5.74 \mathrm{~mm}$, and $h=7 \mathrm{~mm}$.

The simulated results were obtained using HFSS. The experimental results were obtained using a vector network analyzer and broadband horn antennas for the emission and detection of the s- and p-polarized radiation for angles of incidence between 5 and $35^{\circ}$. Figure 2 shows the simulated and measured specular reflection showing an agreement between the two curves. Figure 3 shows the simulated absorption in each cavity, showing that there is a high level of photon sorting and separate absorption.

As was stated before, the use of WCMs to perform photon sorting and detection can be applied to different spectral regions. Of particular interest is photon sorting in the infrared and visible spectral ranges. In the IR, multiband IR detectors can be designed, whereas in the visible and near-IR spectral regions, multi-junction solar cells can be developed. To demonstrate this concept in the IR, we described in [21] the design, fabrication, and testing of IR photon sorting metasurface of a very similar design as the

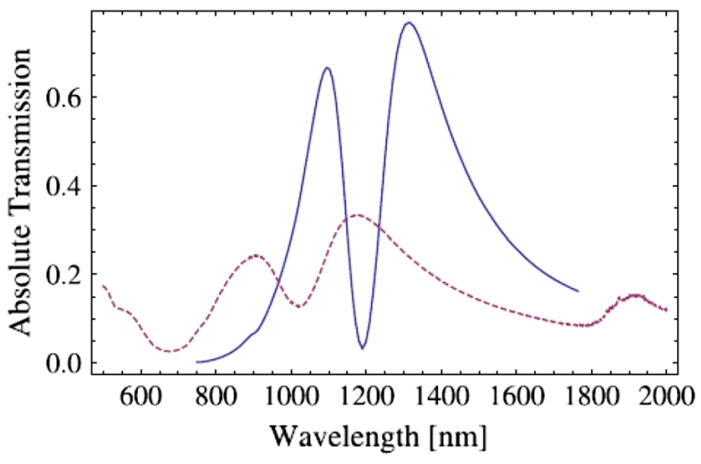

Fig. 5 Simulated (solid blue) and measured (dashed red) absolute transmission. It is seen that the two observed (measured) peaks are shifted and broadened relative to the simulated peaks [21]

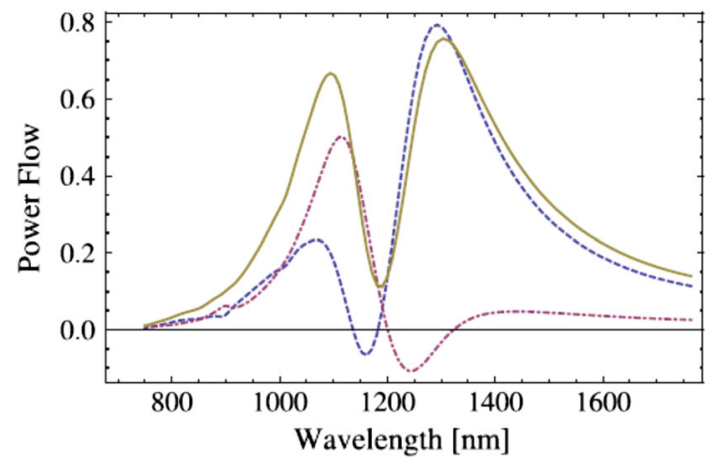

Fig. 6 Simulated power flow through the structure. The smaller (red dot dashed) cavity selects and transmits IR radiation of smaller wavelength $(910 \mathrm{~nm})$ while the larger (blue dashed) cavity selects and transmits IR radiation of larger wavelength $(1,170 \mathrm{~nm})$ [21]

microwave structure shown in Fig. 1, but with the structural feature sizes scaled down considerably: $\Lambda=500 \mathrm{~nm}$, $a_{1}=80 \mathrm{~nm}, a_{2}=125 \mathrm{~nm}, h=35 \mathrm{~nm}$, and with the metal being gold (with the complex permittivity provided by Palik [24]) and amorphous silicon (a-Si) filling the cavities, as shown in Fig. 4b. In this structure, photon sorting and selective transmission through the two differently sized apertures were the objectives. The results of the photon sorting in this higher-energy spectral range (relative to the microwave) are not as dramatic in terms of photon sorting efficiency and agreement between simulated and measured results due to several issues. One issue being the increased optical absorption within the metal. The second issue is the quality of the fabricated structure; better defined and more uniformly shaped cavities can be achieved for the larger size scale microwave structures than for the IR metasurfaces (Figs. 5, 6).

Yet the concept of photon sorting and individual absorption/detection via polarization-independent WCMs (largely polarization independent, see [21] for more discussions) is still evident, and further optimization and 
fabrication process development could provide a compelling way to perform dual-wavelength IR detection in a small, lightweight single-layer metasurface.

\section{Dispersion engineered plasmonic structures}

The ability to custom design the dispersion curves of surface plasmons (SPs) would have great utility in studying complex EM phenomena, including the filtering and trapping of SP modes, and in studying slow- and fast-light phenomena. For such studies, one would like a material that supports SPs that have a dispersion curve that exhibits a zero group velocity or a negative group velocity. Dispersion curves for SPs are dependent on the shape of the metal surface and the overall structure of the metal/ dielectric system. For example, for flat metal surfaces, the SP dispersion curve is given by:

$k_{\mathrm{sp}}=k_{\mathrm{o}}\left(\frac{\varepsilon_{\mathrm{m}} \varepsilon_{\mathrm{d}}}{\varepsilon_{\mathrm{m}}+\varepsilon_{\mathrm{d}}}\right)^{1 / 2}$

where $k_{\mathrm{sp}}$ is the in-plane momentum of the $\mathrm{SP}, k_{\mathrm{o}}=\omega / c$ and $\varepsilon_{\mathrm{m}}$ and $\varepsilon_{\mathrm{d}}$ are the frequency-dependent complex permittivities of the metal and bounding dielectric material, respectively. Equation 2 results in a monotonically increasing dispersion curve for frequencies below $\omega_{\mathrm{p}} / \sqrt{2}$ and for a bounding dielectric of vacuum. Thus, there are no SP momentum ranges where the SP displays a negative or zero group velocity that could be used to trap or concentrate the SPs. There are systems that display negative and/ or zero SP group velocities, including SPs in thin metal films that has symmetric and asymmetric SP modes and SPs on elliptically shaped metallic particles [25-28], yet such systems do not provide the flexibility to "dial-in" the desired SP dispersion curve that one would want in order to study complex SP phenomena.

In [29], we describe a method to engineer SP dispersion curves by using multiple, flat, and unpatterned layers where each layer has a different free electron concentration $n$. Such control of $n$ can be achieved using doped semiconductors and doped oxides [30] and can be used to tune the value of $\varepsilon_{\mathrm{m}}$ for each layer, as described by the Drude model:

$\varepsilon_{\mathrm{m}}=\varepsilon_{\infty}-\frac{\omega_{\mathrm{p}}^{2}}{\omega(\omega+i \Gamma)}$

where $\varepsilon_{\infty}$ is the high-frequency permittivity, $\Gamma=e / m_{\mathrm{eff}} \mu_{\mathrm{e}}$ is the damping rate with $e$ being the electron charge, $m_{\mathrm{eff}}$ and $\mu_{\text {eff }}$ the electron effective mass and mobility, respectively, and with $\omega_{\mathrm{p}}^{2}=n e^{2} / \varepsilon_{o} m_{\mathrm{eff}}$ being dependent on $n$. Thus, each layer can have a different $\varepsilon_{\mathrm{m}}$ and when applying
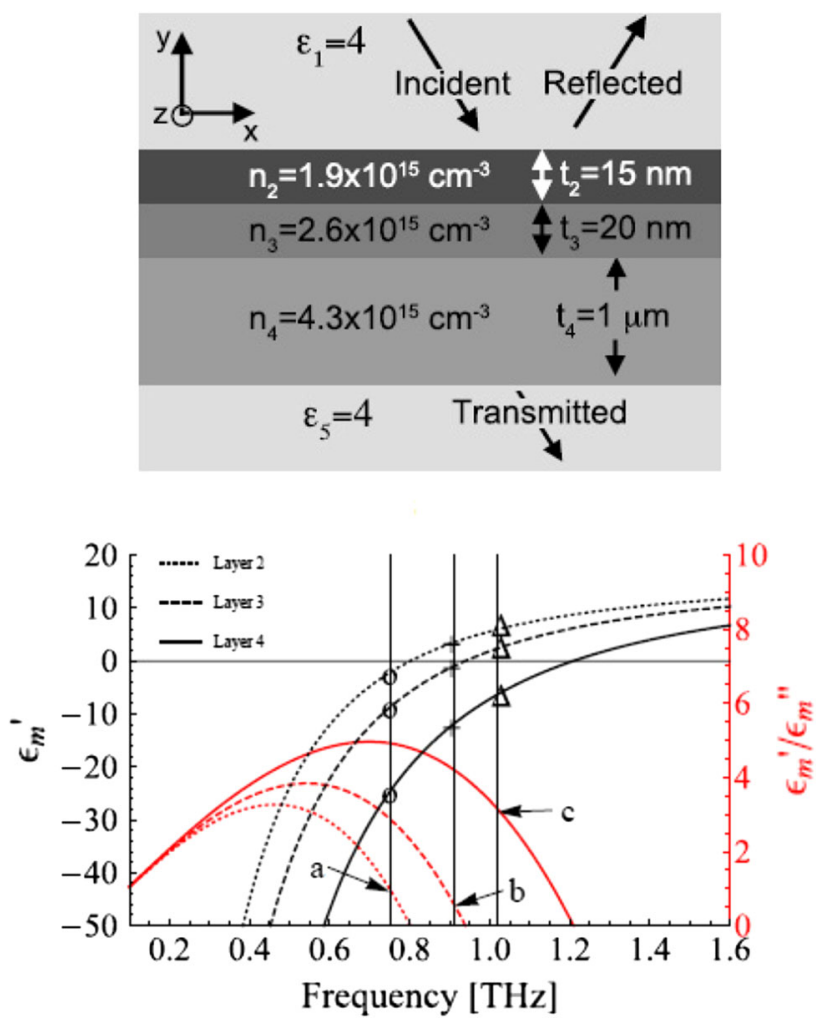

Fig. 7 Top The structure studied in this work and in [29]. Bottom The real part of the permittivity of each layer, and the ratio of the real to the imaginary part

this to Eq. (2) leads to different steps in the dispersion curve given by the equation [29]:

$\omega_{\text {step }, \mathrm{i}}=\frac{\sqrt{\omega_{\mathrm{p}, \mathrm{i}}^{2}-\Gamma^{2}\left(\varepsilon_{\infty}+\varepsilon_{\mathrm{d}}\right) / 4}}{\sqrt{\varepsilon_{\infty}+\varepsilon_{\mathrm{d}}}}$

where the subscript $i$ refers to the layer. This concept relies on how the EM fields of the SP modes change (i.e., distribute themselves or confine themselves) as the in-plane momentum $k_{s p}$ goes from a small value to larger values.

Because of the relationship, $k_{\perp}=i \kappa=\sqrt{\varepsilon_{\mathrm{d}} k_{\mathrm{o}}^{2}-k_{\mathrm{sp}}^{2}}$, where $k_{\perp}$ is the out-of-plane momentum and $\kappa$ is the decay constant, and at low $k_{\mathrm{sp}}$ values, the EM fields of the SP extend to a greater extent throughout all the layers of the structure. But as $k_{\mathrm{sp}}$ increases, so does $\kappa$ and the fields are increasingly confined to evermore higher-lying layers (i.e., layers that are nearer the source that is exciting the SPs).

Thus for low $k_{\mathrm{sp}}$, the energy of the SPs will tend to assume the value of the thickest, bottom most layer, and as $k_{\mathrm{sp}}$ increases, changes in the SP energy will occur as the SP assumes the value appropriate for the layer within which its fields are increasingly confined. Figures 7, 8, 9, and 10 illustrate this concept for a simulated 3-layer system (with two additional semi-infinite layers being the superstrate 

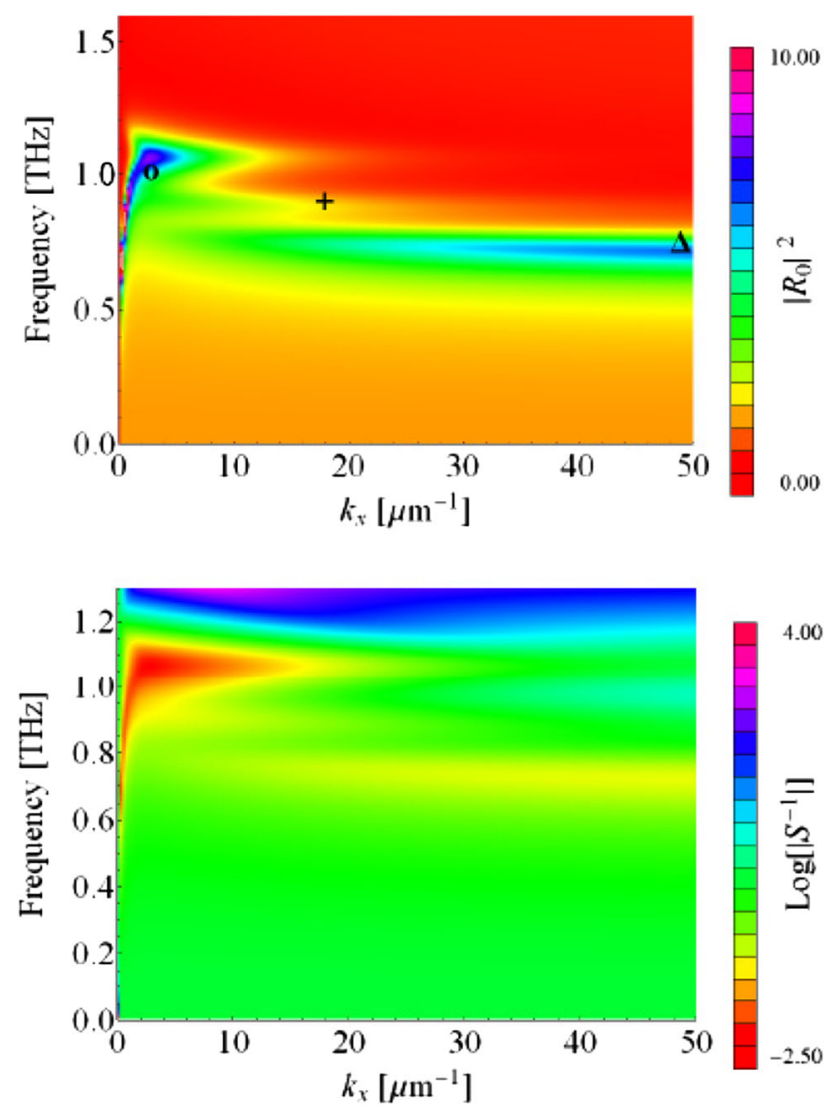

Fig. 8 Top The reflectance (i.e., $|R|^{2}$ ), assuming a unit amplitude plane wave. Of course, for such large $k_{x}$ values, the plane wave is not within the light cone, but such excitation can be achieved with radiating dipoles placed near the surface. Bottom The value of the logarithm of $\left|S^{-1}\right|$. Self-sustaining modes, such as SPs have poles in $\left|S^{-1}\right|[29]$

and substrate of $\varepsilon=4$ ). The structure has 3 layers of different doping, layers 2, 3, and 4. Layer 2 has the following properties: $n_{2}=1.9 \times 10^{15} \mathrm{~cm}^{-3}$ and $t_{2}=15 \mathrm{~nm}$; Layer $3 n_{3}=2.6 \times 10^{15} \mathrm{~cm}^{-3}$ and $t_{3}=20 \mathrm{~nm} ;$ Layer 4

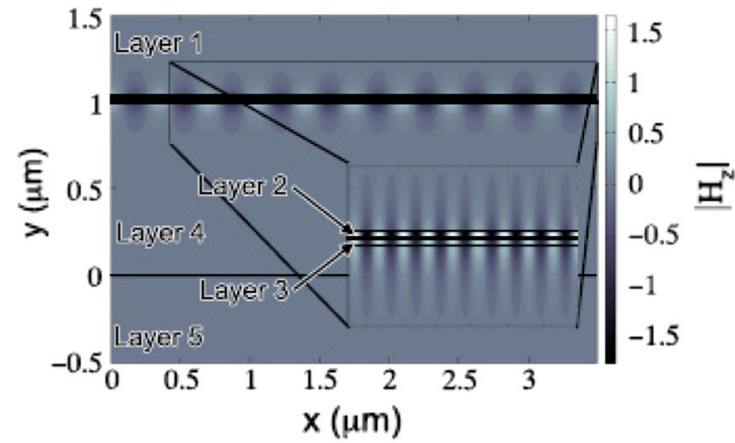

(a)

Fig. 9 Left The value of $\operatorname{Re}\left(H_{z}\right)$ for $v=0.91 \mathrm{THz}$ and $k_{\mathrm{x}}=18 \mu \mathrm{m}^{-1}$ (point "+" in the top figure in Fig. 8). The EM fields of the SP are largely confined to layers 1,2 , and 3. Right The value of $\operatorname{Re}\left(H_{z}\right)$ for

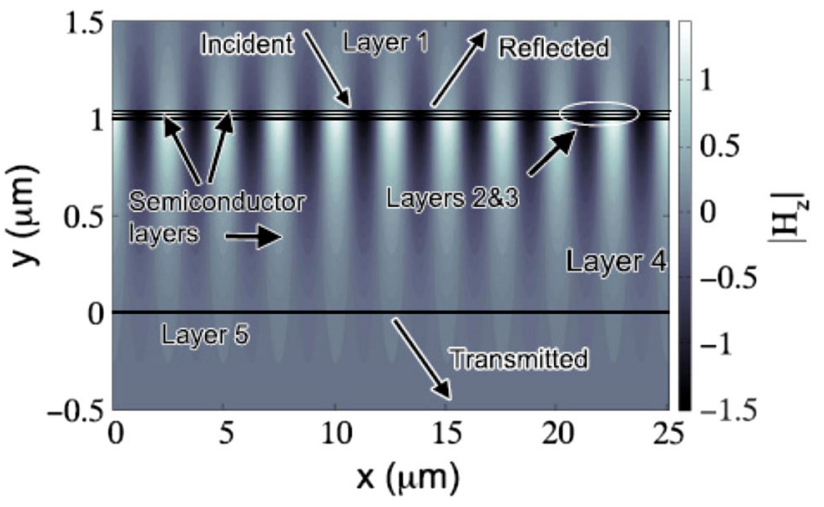

Fig. 10 Value of $\operatorname{Re}\left(H_{\mathrm{z}}\right)$ for $v=1.02 \mathrm{THz}$ and $k_{\mathrm{x}}=2.5 \mu \mathrm{m}^{-1}$ (point "o" in the top figure in Fig. 8). It is seen that the EM fields of the SP for this mode extends throughout the entire structure; thus, the thick Layer 4 will largely determine the energy of this SP mode [29]

$n_{4}=4.3 \times 10^{15} \mathrm{~cm}^{-3}$ and $t_{4}=1 \mu \mathrm{m}$. All layers have $\mu_{\mathrm{e}}=1.5 \times 10^{5} \mathrm{~cm}^{2} / \mathrm{V} \mathrm{s}, m_{\mathrm{eff}}=0.015 m_{\mathrm{o}}$, and $\varepsilon_{\infty}=15.7$. Layers 1 and 5 are considered dielectrics with frequencyindependent permittivity values of $\varepsilon=4$.

With three separate layers, each having different values of $n$ and thus different SP frequencies given by Eq. (4), one would expect 3 different steps in the dispersion curve with the steps at the frequencies given by Eq. (4). Using both a rigorous coupled wave algorithm (RCWA) and finite difference time domain (FDTD) methods (Lumerical), the dispersion curves do indeed show steps or levels with the frequencies predicted by Eq. (4). And by studying the EM field distributions for SP modes at the three different "steps" of the dispersion curve, the distributions agree with what is expected, namely that the EM fields of the SPs for increasing $k_{\mathrm{sp}}$ are increasing confined to the top thinner layers of the structure which impart to the SP a different energy according to the layer's free electron concentration $n$. 


\section{Discussion and conclusions}

Two different concepts involved with sorting and absorbing EM modes were reviewed and described in this work. The first concept involved photon sorting and individual absorption by using compound aperture arrays in metal films. The two differently shaped cavities supported two WCMs of different energy, and as incoming light approaches the film, the photons converge to the cavity that supports excitations that match their energy. Once the WCM is excited, the EM radiation is absorbed and can be used to generate a photocurrent, as would be done in dualwavelength detectors, or to drive electron-hole pair generation, separation and collection, as would be done in a solar cell. The second concept involved the method to engineer the dispersion curves of SPs to produce SP modes that have zero or negative group velocities. The structures required for this are simple and are only flat multi-layered semiconductors of varying free electron charge concentrations. With such a system, SP trapping and filtering can be studied that would include slow- and fast-light phenomena.

Open Access This article is distributed under the terms of the Creative Commons Attribution License which permits any use, distribution, and reproduction in any medium, provided the original author(s) and the source are credited.

\section{References}

1. S. John, Strong localization of photons in certain disordered dielectric superlattices. Phys. Rev. Lett. 58, 2486-2489 (1987)

2. E. Yablonovitch, Inhibited spontaneous emission in solid-state physics and electronics. Phys. Rev. Lett. 58, 2059-2062 (1987)

3. M. Lester, D.C. Skigin, An optical nanoantenna made of plasmonic chain resonators. J. Opt. 13, 035105 (2011)

4. T. Li, S.M. Wang, J.X. Cao, H. Liu, S.N. Zhu, Cavity-involved plasmonic metamaterial for optical polarization conversion. Appl. Phys. Lett. 97, 261113 (2010)

5. R.A. Shelby, D.R. Smith, S.C. Nemat-Nasser, S. Schultz, Microwave transmission through a two-dimensional, isotropic, left-handed metamaterial. Appl. Phys. Lett. 78, 489-491 (2001)

6. R.A. Shelby, D.R. Smith, S. Schultz, Experimental verification of a negative index of refraction. Science 292, 77-79 (2001)

7. D. Schurig, J.J. Mock, B.J. Justice, S.A. Cummer, J.B. Pendry, A.E. Starr, D.R. Smith, Metamaterial electromagnetic cloak at microwave frequencies. Science 314, 977-980 (2006)

8. J. Valentine, J. Li, T. Zentgraf, G. Bartal, X. Zhang, An optical cloak made of dielectrics. Nat. Mater. 8, 568-571 (2009)

9. D. Crouse, Numerical modeling and electromagnetic resonant modes in complex grating structures and optoelectronic device applications. IEEE Trans. Electron Devices 52, 2365-2373 (2005)

10. D. Crouse, A.P. Hibbins, M.J. Lockyear, Tuning the polarization state of enhanced transmission in gratings. Appl. Phys. Lett. 92, 191105 (2008)

11. D. Crouse, E. Jaquay, A. Maikal, A.P. Hibbins, Light circulation and weaving in periodically patterned structures. Phys. Rev. B 77, 195437 (2008)
12. D. Crouse, P. Keshavareddy, Polarization independent enhanced optical transmission in one-dimensional gratings and device applications. Opt. Express 15, 1415-1427 (2007)

13. D. Crouse, P. Keshavareddy, A method for designing electromagnetic resonance enhanced silicon-on-insulator metal-semiconductor-metal photodetectors. J. Opt. 8, 175-181 (2006)

14. I. Mandel, J.N. Gollub, I. Bendoym, D.T. Crouse, Theory and Design of a Novel Integrated Polarimetric Sensor Utilizing a Light Sorting Metamaterial Grating. Sens. J. IEEE 13(2), 618-625 (2013)

15. I. Mandel, E. Lansey, J.N. Gollub, D.T. Crouse, An effective cavity resonance model for enhanced optical transmission through a periodic array of subwavelength square apertures. In SPIE NanoScience + Engineering . International Society for Optics and Photonics. (2012), pp. 845735-845735

16. I. Bendoym, A.B. Golovin, D.T. Crouse, The light filtering and guiding properties of high finesse phase resonant compound gratings. Opt. Express 20(20), 22830-22846 (2012)

17. E. Lansey, N. Pishbin, J.N. Gollub, D.T. Crouse, Analytical analysis of the resonance response of subwavelength nanoscale cylindrical apertures in metal at near-ultraviolet, optical, and near-infrared frequencies. JOSA B 29(3), 262-267 (2012)

18. A. Enemuo, M. Nolan, Y.U. Jung, A.B. Golovin, D.T. Crouse, Extraordinary light circulation and concentration of s-and p-polarized phase resonances. J. Appl. Phys. 113(1), 014907 (2013)

19. D.T. Crouse, Y.H. Lo, Nonsteady-state surface plasmons in periodically patterned structures. J. Appl. Phys. 95(8), 4163-4172 (2004)

20. N. Yu, P. Genevet, M.A. Kats, F. Aieta, J.P. Tetienne, F. Capasso, Z. Gaburro, Light propagation with phase discontinuities: generalized laws of reflection and refraction. Science 334(6054), 333-337 (2011)

21. I.M. Mandel, E. Lansey, J.N. Gollub, C.H. Sarantos, R. Akhmechet, A.B. Golovin, D.T. Crouse, Photon sorting in the near field using subwavelength cavity arrays in the near infrared. Appl. Phys. Lett. 103(25), 251116 (2013)

22. E. Lansey, I.R. Hooper, J.N. Gollub, A.P. Hibbins, D.T. Crouse, Light localization, photon sorting, and enhanced absorption in subwavelength cavity arrays. Opt. Express 20(22), 24226-24236 (2012)

23. S. Herminghaus, M. Klopfleisch, H.J. Schmidt, Attenuated total reflectance as a quantum interference phenomenon. Opt. Lett. 19, 293-295 (1994)

24. E.D. Palik, Handbook of Optical Constants of Solids (Academic Press, London, 1985)

25. H. Raether, surface plasmons on smooth and rough surfaces and on gratings, vol. 11 of springer tracts in modern physics (Springer, Berlin, 1988)

26. R. Zia, M.D. Selker, P.B. Catrysse, M.L. Brongersma, Geometries and materials for subwavelength surface plasmon modes. J. Opt. Soc. Am. 21, 2442-2446 (2004)

27. V. Chegel, Y. Demidenko, V. Lozovski, A. Tsykhonya, Influence of the shape of the particles covering the metal surface on the dispersion relations of surface plasmons. Surf. Sci. 602, 1540-1546 (2008)

28. P. Yeh, Optical waves in layered media, vol. 95 of Wiley series in pure and applied optics (Wiley, London, 1988)

29. I.M. Mandel, I. Bendoym, Y.U. Jung, A.B. Golovin, D.T. Crouse, Dispersion engineering of surface plasmons. Opt. Express 21(26), 31883-31893 (2013)

30. P. West, S. Ishii, G. Naik, N. Emani, V. Shalaev, A. Boltasseva, Searching for better plasmonic materials. Laser Photon. Rev. 4, 795-808 (2010) 